\title{
MATEMATICA E CALCOLO SCIENTIFICO
}

\author{
L. DONATELLA MARINI (*)
}

Nota presentata dal m.e. Piero Colli Franzone

(Adunanza del 28 marzo 2019)

SuNTO. - In questa breve comunicazione cercherò di dare un'idea di cosa si intenda per Calcolo Scientifico e di quale sia il ruolo della Matematica all'interno di esso. Illustrerò su un esempio estremamente semplice le fasi che portano dalla modellizzazione di un fenomeno fisico alla sua risoluzione. Elencherò vari problemi di interesse per le applicazioni, limitandomi a quelli più vicini alle mie competenze, e mostrerò le difficoltà che possono insorgere presentando casi famosi di "fallimenti" dovuti a piccoli, apparentemente trascurabili, errori in una qualunque delle fasi.

$* * *$

ABSTRACT. - This short note is meant to give an idea of what Scientific Computing is and what its goal is. In particular, I will focus on the role of Mathematics inside Scientific Computing. On an extremely simple example I will indicate the various steps that lead from the model of the physical phenomenon to the practical solution. I will provide a list of few problems of interest for the applications, among those closer to my field of expertise, and finally I will show some famous cases of failure due to small, almost negligible, errors in one of the steps.

\section{INTRODUZIONE}

In questa breve nota cercherò di spiegare, seppure in modo inevitabilmente approssimativo, cosa si intende per Calcolo Scientifico e soprat-

(*) Professore Emerito Università degli Studi di Pavia, Dipartimento di Matematica, Pavia, Italy. E-mail: donatella.marini@unipv.it - marini@imati.cnr.it 
tutto quale ruolo svolge all'interno di esso la Matematica cosiddetta Applicata. Sottolineo "cosiddetta", in quanto il termine Matematica Applicata, in opposizione a Matematica Pura, è discusso e criticato da molti, non senza valide ragioni: la Matematica è una sola, e tutta è applicabile. Sarebbe più appropriata la dicitura Matematica per le applicazioni.

Sotto il nome "Calcolo Scientifico" si colloca tutta una serie di attività di ricerca che vede coinvolti ricercatori provenienti da ambiti diversi, con competenze in diversi settori scientifici, allo scopo di predire l'andamento di fenomeni fisici di interesse applicativo tramite la simulazione numerica, come supporto alle scelte industriali e, più in generale, alle decisioni da prendere.

Molto grossolanamente, il Calcolo Scientifico si pùo schematizzare in quattro fasi di intervento. Nella prima fase viene individuato il problema fisico di interesse. Gli esempi di problemi da trattare sono numerosissimi, e vanno dalla Fisica alla Chimica, ai più svariati aspetti dell'Ingegneria, e più recentemente alla Biologia, all'Economia, alla Medicina, eccetera. Nella seconda fase si deve passare dal problema fisico alla sua modellizzazione: il problema va tradotto in opportune equazioni che descrivano in maniera esaustiva il fenomeno che si deve analizzare. Si passa poi alla fase di risoluzione delle equazioni descrittive del fenomeno e infine alla rappresentazione e interpretazione dei risultati.

Problema fisico $\rightarrow$ Modello $\longrightarrow$ Approssimazione $\rightarrow$ Risoluzione e interpretazione

\section{DAL PROBLEMA Fisico ALLA SUA RISOLUZIONE}

Come dicevo, i problemi di interesse nelle applicazioni sono numerosissimi. Ne citerò solo alcuni, più legati alla tipologia delle mie ricerche: uno dei primi settori nei quali si è sviluppata la simulazione numerica (tralasciando le applicazioni belliche, alle quali non ho mai partecipato) è sicuramente quello della progettazione degli aerei da trasporto civile, per combinare costi (di costruzione e di consumo) e sicurezza. In particolare ci si deve muovere in ambiti ingegneristici molto diversi tra loro, come la dinamica dei fluidi, la dinamica delle strutture, l'elettronica, la combustione, l'acustica, eccetera. Questo, in generale, viene trattato da gruppi diversi (ciascuno con le proprie competenze specifiche), che vanno periodicamente messi in cantatto. Un altro settore (per alcuni versi simile) che 
ha visto un enorme sviluppo è quello automobilistico, per combinare prestazioni, comodità, sicurezza ed estetica. Le applicazioni legate in qualche modo alla geologia hanno avuto anch'esse sviluppi enormi: dalla ottimizzazione dello sfruttamento dei pozzi petroliferi allo studio delle catastrofi naturali (terremoti, inondazioni, etc.) senza tralasciare la gestione delle scorie (da quelle radioattive alla più comune plastica).

In tempi più recenti si è avuto un grandissimo sviluppo delle applicazioni alla biologia e alla medicina, dove il problema di "descrivere matematicamente cosa succede nei vari casi” risulta molto più difficile che nei casi precedenti (che pure non sono facili).

Un altro settore che ha visto sviluppi enormi in tempi recentissimi è poi quello della intelligenza artificiale, che però, al momento, è molto più vicino all'informatica che al calcolo scientifico.

Per evitare di entrare in dettagli troppo tecnici, che tra l'altro richiederebbero competenze che, come praticamente avviene in tutti i settori, si avviano verso forme di specializzazione sempre più spinte, mi limiterò a pochi accenni superficiali a qualche caso molto particolare.

\subsection{Alcuni esempi di modelli matematici}

Dato il problema di interesse, per poterlo studiare lo si deve descrivere in maniera corretta tramite un modello opportuno. Questo avviene nella seconda fase: il problema va tradotto in opportune equazioni che descrivano in maniera sufficientemente accurata il fenomeno che si deve analizzare. Nella grande maggioranza dei casi si tratta di equazioni differenziali, ossia equazioni in cui compaiono delle derivate. Un esempio è rappresentato dalle equazioni di Navier-Stokes per fluidi incomprimibili (come l'acqua e l'aria), che sono alla base di tutti i problemi di Fluidodinamica

$$
\left\{\begin{array}{l}
\frac{\partial u}{\partial t}-\nu\left(\frac{\partial^{2} u}{\partial x^{2}}+\frac{\partial^{2} u}{\partial y^{2}}+\frac{\partial^{2} u}{\partial z^{2}}\right)+u \frac{\partial u}{\partial x}+v \frac{\partial u}{\partial y}+w \frac{\partial u}{\partial z}+\frac{1}{\rho} \frac{\partial p}{\partial x}=F_{x} \\
\frac{\partial v}{\partial t}-\nu\left(\frac{\partial^{2} v}{\partial x^{2}}+\frac{\partial^{2} v}{\partial y^{2}}+\frac{\partial^{2} v}{\partial z^{2}}\right)+u \frac{\partial v}{\partial x}+v \frac{\partial v}{\partial y}+w \frac{\partial v}{\partial z}+\frac{1}{\rho} \frac{\partial p}{\partial y}=F_{y} \\
\frac{\partial w}{\partial t}-\nu\left(\frac{\partial^{2} w}{\partial x^{2}}+\frac{\partial^{2} w}{\partial y^{2}}+\frac{\partial^{2} w}{\partial z^{2}}\right)+u \frac{\partial w}{\partial x}+v \frac{\partial w}{\partial y}+w \frac{\partial w}{\partial z}+\frac{1}{\rho} \frac{\partial p}{\partial z}=F_{z} \\
\frac{\partial u}{\partial x}+\frac{\partial v}{\partial y}+\frac{\partial w}{\partial z}=0
\end{array}\right.
$$

Si tratta di un sistema di 4 equazioni differenziali alle derivate par- 
ziali nello spazio e nel tempo per le 4 incognite: $u, v, w$ (le tre componenti della velocità) e $p$ (la pressione). I dati del problema sono le componenti della forza applicata $F_{x}, F_{y}, F_{z}$, e i coefficienti $\rho$ e $v$, rispettivamente densità e viscosità del fluido. Sono equazioni molto complicate da trattare e, anche se a noi Matematici piace scriverle nella forma più compatta

$$
\left\{\begin{array}{l}
\frac{\partial \mathbf{U}}{\partial t}-\nu \Delta \mathbf{U}+\mathbf{U} \cdot \nabla \mathbf{U}+\frac{1}{\rho} \nabla p=\mathbf{F} \\
\nabla \cdot \mathbf{U}=0
\end{array}\right.
$$

non cambia la sostanza: si guadagna in eleganza ma le difficoltà restano ovviamente le stesse.

Un altro esempio è costitutito dalle equazioni di Maxwell, che modellizzano i fenomeni di elettro-magnetismo. Nel vuoto si scrivono così

$$
\left\{\begin{array}{l}
\varepsilon_{0} \nabla \cdot \boldsymbol{E}=\rho \\
\frac{\partial \boldsymbol{B}}{\partial t}+\nabla \times \boldsymbol{E}=0 \\
\nabla \cdot \boldsymbol{B}=0 \\
\varepsilon_{0} \mu_{0} \frac{\partial \boldsymbol{E}}{\partial t}-\nabla \times \boldsymbol{B}=\mu_{0} \boldsymbol{J}
\end{array}\right.
$$

Le quantità incognite sono il campo elettrico $E$ e il campo di induzione magnetica $B$. Gli altri parametri che compaiono nelle equazioni sono dati, ossia sono quantità note: $\varepsilon_{0}$ e $\mu_{0}$ sono rispettivamente la permittività elettrica e magnetica, $\rho$ e $J$ sono la densità di carica e di corrente rispettivamente.

Un altro esempio è l'equazione di Schr"odinger, fondamentale in meccanica quantistica, che determina l'evoluzione nel tempo dello stato di un sistema, ad esempio di un elettrone o di una particella subatomica nello spazio e nel tempo sotto l'azione di varie forze:

$$
i \hbar \frac{\partial}{\partial t} \Psi(\mathbf{r}, t)=\widehat{H} \Psi(\mathbf{r}, t)
$$

Una descrizione delle quantità in gioco che possa essere chiara per non addetti ai lavori è praticamente impossibile: l'incognita è la funzione d'onda $\Psi, i$ è l'unità immaginaria, $\hbar$ la costante di Planck e $\hat{H}$ l'operatore Hamiltoniano. 
È evidente che si tratta di equazioni molto complesse, impossibili da risolvere con carta e penna. E come già osservato da Fourier, un'equazione, per quanto bella, è di scarsa utilità se non la si sa risolvere, almeno in modo approssimato. Bisogna allora cercare di trovare soluzioni approssimate di queste equazioni, individuando dei metodi opportuni che possano essere implementati su computers e diano informazioni il più possibile accurate del fenomeno che si sta studiando. Naturalmente l'attendibilità dei metodi utilizzati viene filtrata dall'esperienza dell'utilizzatore (Ingegnere, Fisico, Chimico eccetera) e dallo studio teorico del Matematico.

Si passa quindi alla terza fase, ossia alla approssimazione numerica, e successivamente alla rappresentazione e interpretazione dei risultati ottenuti.

\subsection{Risoluzione numerica e visualizzazione dei risultati}

Non essendo in grado di ottenere un'espressione analitica della soluzione esatta, la soluzione viene calcolata, in modo approssimato, in alcuni punti del dominio del problema.

Per dare un'idea di come si procede nella risoluzione numerica considererò come esempio un problema particolarmente semplice, accessibile anche a chi non ha dimestichezza con la matematica. Immaginiamo di volere calcolare e visualizzare la distribuzione della temperatura in una stanza, che per semplicità assumiamo bidimensionale e quadrata. Su una parete si trova una porta-finestra a zero gradi, su un'altra parete un termosifone a 50 gradi, e le restanti pareti sono ben coimbentate, in modo che non ci sia dispersione di calore (Fig. 1)

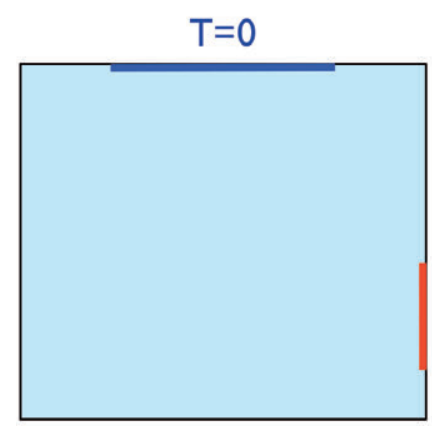

Fig. 1.

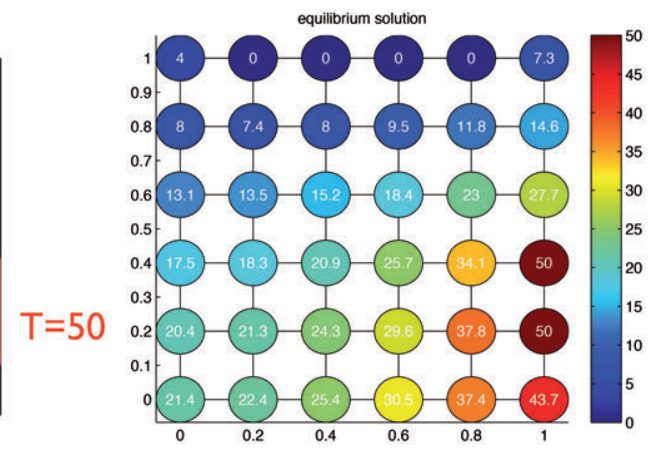

Fig. 2. 
A regime, cioè dopo che la distribuzione del calore si è assestata e non varia più, la temperatura $T$ è soluzione del seguente problema differenziale

$$
\left\{\begin{array}{lr}
\Delta T=0 & \text { all'interno della stanza } \\
T=0 & \text { in corrispondenza della porta-finestra } \\
T=50 & \text { in corrispondenza del termosifone } \\
\frac{\partial T}{\partial n}=0 & \text { lungo le restanti pareti }
\end{array}\right.
$$

La lettera greca $\Delta$ indica l'operatore di Laplace, cioè la somma delle derivate seconde rispetto alle variabili spaziali, nel nostro caso $x$ e $y$ : $\Delta T=\frac{\partial^{2} T}{\partial x^{2}}+\frac{\partial^{2} T}{\partial y^{2}}$.



Fig. 3.

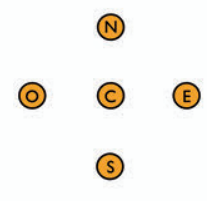

Nonostante si tratti di un problema molto semplice, non siamo in grado di risolverlo in modo esatto, e ci accontentiamo di calcolare la soluzione in modo approssimato. Il metodo numerico più antico, nonch e il più semplice da spiegare, è il cosiddetto metodo delle differenze finite: dopo avere suddiviso la stanza con una griglia di quadrati, in ogni punto interno della griglia si approssimano le derivate seconde con opportune differenze dei valori della temperatura nei punti vicini (Fig. 3).

Nel caso dell'esempio, assumendo per semplicità che la distanza tra i punti della griglia sia pari a 1 , questo equivale ad approssimare la soluzione in ogni punto interno come media dei valori nei quattro punti vicini (Nord, Sud, Est e Ovest): 


$$
T^{C}=\frac{T^{N}+T^{S}+T^{E}+T^{O}}{4}
$$

Nei punti lungo il termosifone e la porta-finestra la temperatura è nota e data da $T=50$ e $T=0$ rispettivamente. Nei restanti punti lungo il bordo della stanza si pùo imporre che il valore di $T$ sia uguale al valore nel punto interno più vicino. In questo modo si scrive un sistema di 36 equazioni che viene risolto su computer. Il risultato fornito dal computer è una lista di numeri (in questo caso 36, uno per ogni punto della griglia) che rappresentano i valori della temperatura nei 36 punti della griglia e che vanno opportunamente visualizzati. Un esempio di rappresentazione è quello della Fig. 2, ma chiaramente non fornisce un'idea accurata di come si distribuisca la temperatura. Una rappresentazione come quella della Fig. 4 sembra essere migliore per cui, procedendo in questo modo e suddividendo in griglie sempre più fitte si arriva ad una rappresentazione molto chiara, come si vede nella Fig. 5. Lo scopo di questo semplice esempio è duplice: da una parte sottolineare come la scelta del metodo numerico per approssimare la soluzione sia importante per ottenere risultati con un elevato grado di accuratezza, dall'altra mostrare come la rappresentazione dei risultati sia anch'essa pure di fondamentale importanza per una corretta lettura dei risultati stessi e conseguente comprensione del fenomeno fisico.

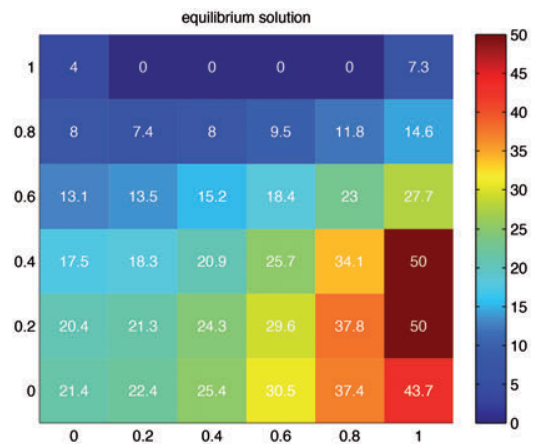

Fig. 4.

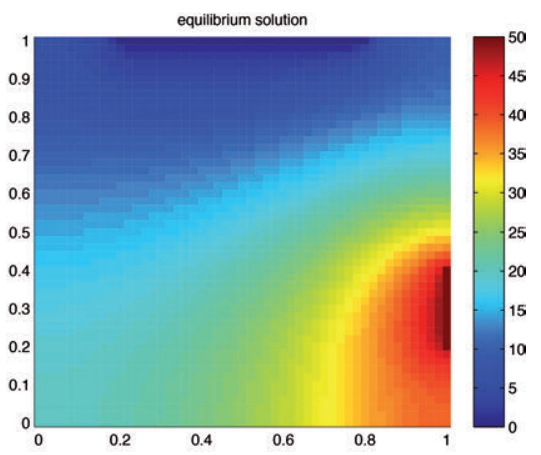

Fig. 5.

Nel caso dell'esempio appena descritto le varie fasi sono ben individuate ed in sequenza: modello, approssimazione numerica, risoluzione e visualizzazione dei risultati. In generale però, come si pùo facilmente intuire, le nostre fasi non vengono portate avanti in successione (come le 
ho accennate), ma con un continuo va e vieni, dove gli esiti e le difficoltà nelle fasi seguent $i$ inducono a ritoccare (a volte in qualche dettaglio, altre volte in modo sostanziale) la formulazione e il trattamento delle fasi precedenti. Se vi è mai accaduto di vedere all'opera un gruppo di formiche (o di api, o di molti altri animali) sappiate che la stessa apparente confusione e lo stesso disordine avviene, molto spesso, anche nella ricerca scientifica. Così come, spessisimo, in entrambi i casi il risultato finale è di ottima qualità (potenza del disordine..).

Ma attenzione: praticamente sempre le equazioni differenziali e/o i modelli su cui lavorano i matematici dipendono in generale da tutta una quantità di coefficienti (le proprietà dei materiali, le condizioni dell'ambiente, etc.) che spesso sono noti solo in modo approssimato, e conseguentemente le loro soluzioni dipendono anch'esse da questi coefficienti.

Se non si conoscono abbastanza bene alcuni coefficienti si risolve, sempre in modo approssimato, il problema per diversi valori dei coefficienti, scegliendo casi particolari in cui siamo in grado (as esempio, in un esperimento di laboratotio controllato) di misurare il comportamento della soluzione esatta. Se la soluzione numerica e la misurazione in laboratorio non sono abbastanza vicine vorrà dire che la risoluzione numerica è stata fatta male (come sostiene il matematico modellista) oppure che la equazione da risolvere non descrive adeguatamente il fenomeno (come sostiene il matematico numerista). A volte i due si mettono d'accordo, e se la prendono con chi ha preparato l'esperimento....

L'esempio delle formiche comincia a sembrare meno paradossale...

Ma c'è di peggio. In altri casi, ancora più difficili, la mancata conoscenza dei coefficienti pùo essere drammatica e (dal punto di vista scientifico), quasi insormontabile. Un chiaro esempio di una situazione di questo genere l'abbiamo, purtroppo, di fronte proprio in questi giorni: la propagazione, in una popolazione, di un virus. Non è molto difficile scrivere equazioni (in fondo abbastanza semplici) che predicano la probabile evoluzione del numero di contagiati da un giorno all'altro. Purtroppo, la semplicità di tali equazioni viene pagata con l'incertezza sui coefficienti. Conoscendo, oggi, il numero di contagiati (sintomatici e asintomatici), potremmo predire abbastanza facilmente il numero probabile di contagiati domani se solo sapessimo: la probabilità media di guarigione, la probabilità media di decesso, la frequenza media dei contatti, la percentuale di popolazione immune, e la probabilità che un contatto tra un contagiato e un "non immune" dia origine a un nuovo contagio... Insomma: basterebbe conoscere una manciata di coefficienti e sapremmo fare delle 
previsioni abbastanza accurate. Purtroppo questi stessi coefficienti non sono facili da stimare, e inoltre variano nel tempo e nello spazio. Inoltre, il numero stesso di contagiati (sintomatici e asintomatici) per ogni giorno passato è conosciuto solo in modo molto approssimativo. Quello che si pùo tentare di fare (e che viene tentato) è di procedere per tentativi ed errori rifacendosi ai risultati precedenti: partendo (con notevole ottimismo) dalla conoscenza del numero di contagiati a una certa data "D", si possono fare ipotesi approssimative sui valori dei coefficienti, e simulare (con le nostre equazioni) l'evoluzione del fenomeno: se i risultati delle equazioni collimano con le misure fatte nei giorni successivi a "D", possiamo pensare che la nostra stima dei coefficienti sia corretta. Altrimenti si ripete il calcolo con una stima differente. La bravura consiste nel capire (in base al confronto tra la stima precedente e la misura) in che direzione correggere i coefficienti per avere un risultato migliore: per niente facile!

\subsection{Cenni ad altri metodi numerici}

In molti casi pùo risultare complicato usare reticoli regolari come quello della Fig. 3. Per esempio, quando il dominio computazionale presenta una geometria complessa (come il telaio di un'automobile o il motore di un aereo), o il problema considerato ha coefficienti con brusche variazioni, o quando l'accuratezza richiesta alla soluzione non è omogenea sul dominio (in un crash test su un autoveicolo, l'accuratezza richiesta è maggiore in prossimit della zona di impatto). Una griglia di triangoli o quadrilateri (tetraedri o esaedri in 3 dimensioni) si presta meglio a seguire le possibili oscillazioni e/o geometrie complicate. Nella Fig. 6 si vede un esempio molto semplice di una griglia bidimensionale; i pallini rossi sono i punti del domnio in corrispondenza ai quali verrà calcolata la soluzione approssimata. La Fig. 7 mostra invece un esempio di una griglia tridimensionale per la discretizzazione dell'ala di un aereo.

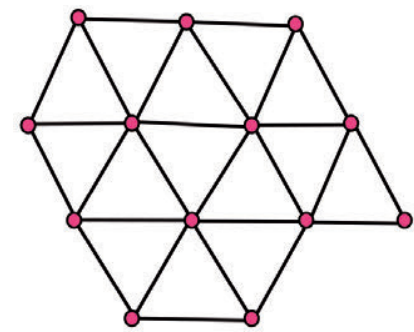

Fig. 6. Esempio di mesh.

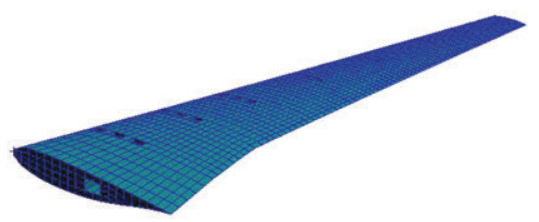

Fig. 7. Mesh per ala di aereo. 
Si sono allora cercati altri metodi in alternativa alle differenze finite che permettessero di ampliare il campo di applicazioni e i problemi da trattare. Tra questi va certamente menzionato il metodo cosiddetto degli Elementi Finiti. Introdotto negli anni sessanta del secolo scorso, mantiene una posizione dominante nel panorama delle tecniche numeriche di approssimazione ed è tuttora il metodo largamente più diffuso e utilizzato nei codici sia di ricerca che industriali. Senza entrare nei dettagli, il metodo non si limita a calcolare solo i valori della soluzione approssimata nei nodi della griglia ma fornisce una soluzione approssimata come superficie polinomiale a tratti.

In tempi più recenti si sono esplorati nuovi metodi di approssimazione in cui, partendo da un approccio e una formulazione simili a quelli del metodo degli elementi finiti, si potessero usare decomposizioni in poligoni (o poliedri in tre dimensioni) di forma arbitraria. Tra i vari approcci mi limito a menzionare, per motivi di puro orgoglio, il metodo introdotto dal gruppo di ricerca di cui faccio parte, che abbiamo chiamato Metodo degli Elementi Virtuali, e che sta riscuotendo molto successo nella comunità scientifica internazionale. Le motivazioni che ci hanno spinto in questa direzione sono essenzialmente due. Da una parte griglie poligonali e poliedriche sono molto facili da generare con programmi esistenti. Dall'altra, in molte applicazioni il loro uso sembra essere la cosa giusta da fare, soprattutto quando si ha a che fare con materiali eterogenei e irregolari. Prendiamo ad esempio il problema dello sfruttamento dei giacimenti petroliferi (Fig. 8).

Dopo che il primo gigantesco getto di petrolio è fuoriuscito dal terreno, solo circa il $5 \%$ del petrolio presente in profondità è stato liberato e bisogna recuperare il restante $95 \%$. Il modo di procedere attualmente in uso è il seguente: si inietta nel terreno un liquido (tipicamente acqua, in una prima fase) che deve spingere il petrolio verso il pozzo di raccolta. Se per esempio la pressione del liquido iniettato è troppo alta, si rischia di recuperare solo acqua. Se è troppo bassa, si recupera il petrolio a ritmi troppo lenti. Da cui l'importanza della simulazione numerica del fenomeno, che a sua volta ha come stadio iniziale la rappresentazione numerica del terreno, fatto di rocce di dimensioni e forme differenti che vanno ovviamente prese in considerazione con un livello sufficiente di dettaglio (e una mesh di poliedri ad-hoc opportunamente scelta pùo rivelarsi molto utile). La simulazione numerica di giacimenti petroliferi è uno dei molti problemi realistici per i quali il Matematico applicato (e in particolare l'Analista Numerico) pùo fornire, e di fatto fornisce, competenze utili per individuare il metodo più appropriato per la risoluzione numerica. La Fig. 9 si riferisce al problema 


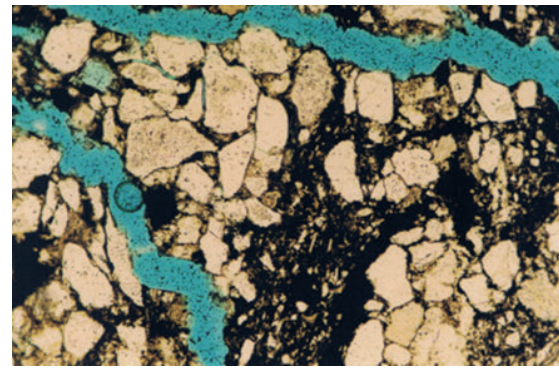

Fig. 8. Giacimento petrolifero.

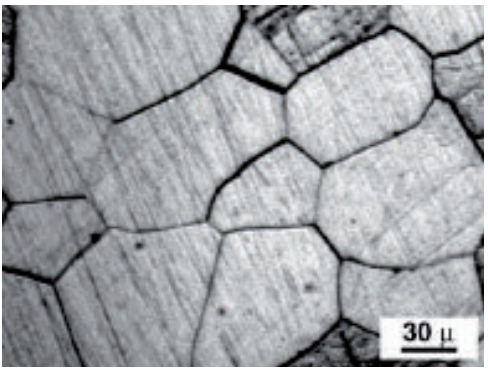

Fig. 9. Crescita di cristalli.

di studiare la crescita di cristalli. Ho volutamente accostato le Figg. 8 e 9 per mostrare problemi in cui i domini computazionali hanno dimensioni molto diverse, di vari ordini di grandezza: dai chilometri cubici di un giacimento petrolifero a poche centinaia di micron cubici nel caso dei cristalli, ma i due problemi presentano strutture molto simili e in entrambi i casi per ottenere una accuratezza accettabile servono decomposizioni adeguate. I campi di applicazione di tutti questi metodi sono svariati e mi limito ad alcuni esempi a carattere indicativo: lo studio del flusso attorno ad un ostacolo (ad esempio l'aria attorno allo spoiler di un auto), oppure del flusso sanguigno attraverso la valvola aortica, e per problemi di questo e di altro tipo lo studio e lo sviluppo di metodi per raffinare la mesh in modo automatico in funzione della soluzione. Questo si rende necessario in problemi in cui la soluzione presenta dei bruschi cambiamenti, come avviene ad esempio per la pressione dell'aria sull'ala di un aereo: per passi successivi e tramite algoritmi opportuni si individuano le zone dove la soluzione numerica passa da valori bassi a valori alti e si infittisce la mesh solo in queste zone.

Passo ora a mostrare alcuni casi di fallimenti, dovuti a piccoli errori in una qualunque delle fasi, per far capire quanto possa essere delicato il calcolo scientifico.

\section{NON SEMPRE VA TUTTO BENE}

Nel corso degli anni si sono purtroppo verificati anche fallimenti, che in alcuni casi hanno provocato disastri economici non indifferenti. Quasi sempre i fallimenti sono dovuti a piccoli errori, all'apparenza trascurabili, come negli esempi che vado a mostrare. 


\subsection{Un errore nel modello: il crollo del Tacoma bridge}

Costruito tra il 1938 e il 1940 (senza computers), il ponte sospeso sul fiume Tacoma nello stato di Washington era un gioiello architettonico, il terzo ponte sospeso più lungo al mondo dopo il Golden Gate e il George Washington. Da subito il ponte mostrò tendenza ad oscillazioni verticali in presenza di forti venti, che tuttavia non destarono troppa preoccupazione, anzi furono da alcuni interpretate come indice di una buona elasticità. Dopo soli 4 mesi dall'inaugurazione il ponte fu investito da raffiche di vento a 42 miglia orarie, iniziò a torcersi e a produrre oscillazioni sempre più ampie fino a che, dopo un'ora e 10 minuti, cedette e crollò. La commissione di inchiesta non arrivò a formulare una causa precisa ma una serie di ipotesi di lavoro. Fu un ingegnere italiano, Giulio Krall, il primo a indicare una spiegazione tecnica del crollo. I calcoli da lui effettuati stabilirono che la velocità critica del vento per il raggiungimento del punto di rottura della struttura dovesse essere di $67 \mathrm{~km} / \mathrm{h}$, praticamente quasi coincidente con l'effettiva velocità del vento di quel giorno. Il progetto non aveva previsto che si potesse verificare una situazione così poco probabile di un vento che potesse entrare in risonanza con la frequenza di vibrazione della struttura. Fortunatamente non ci furono vittime, tranne un cane intrappolato in un auto e talmente terrorizzato da rendere impossibile l'intervento dei soccorritori.

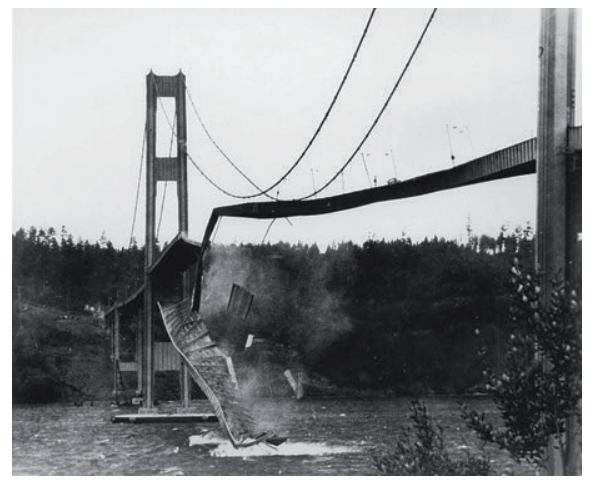

Fig. 10. Crollo del Tacoma.

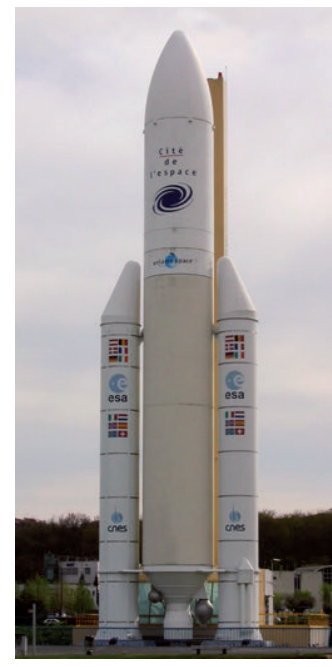

Fig. 11. Ariane 5. 


\subsection{Un errore di software: il disastro di Ariane 5}

Il 4 giugno 1996 il vettore Ariane 5, progetto della Agenzia Spaziale Europea costato 10 anni di lavoro e 7 miliardi di dollari, fu lanciato nelle spazio e fatto esplodere dopo circa 40 secondi dal lancio per evitare che precipitasse a terra a causa di un malfunzionamento. Fu aperta un'inchiesta per individuare le cause del fallimento e dopo due settimane di lavoro la commissione preposta giunse alla conclusione che si era trattato di un errore di software, e precisamente di una erronea rappresentazione dei numeri nel computer. Il software di Ariane 5 era una modifica di quello relativo al modello precedente, Ariane 4. Nel processo di conversione un indicatore di velocità fu convertito da una rappresentazione decimale a 64 bit in una intera a 16 bit con segno: il numero era più grosso di 32.767 , cioè del massimo numero rappresentabile come intero a 16 bit con segno e mandò in tilt il sistema. Ariane ricevette il comando di procedere con velocità negativa ed iniziò a puntare verso la terra. Per evitare danni maggiori la navetta fu fatta esplodere e questo pose fine a molte delle ambizioni spaziali europee.

\subsection{Un errore nella approssimazione numerica}

Si tratta del crollo dello Sleipner-A, una piattaforma petrolifera al largo delle coste della Norvegia: una costruzione gigantesca che poggiava su 24 pilastri piantati a circa 82 metri sul fondo marino. Il ponte superiore, con un'area di 16.000 metri quadri, pesava circa 57.000 tonnellate ed era in grado di ospitare 200 persone con un equipaggiamento di attrezzi per gli scavi di 40.000 tonnellate. Nella simulazione numerica ad elementi finiti con il codice NASTRAN, all'epoca il miglior codice esistente, la sezione circolare dei pilastri fu approssimata con una poligonale, e l'interstizio fra i pilastri (cosiddetto tri-cell) divenne un solo triangolo della griglia. All'epoca, nel 1991, i computers non erano potenti come quelli attuali, e l'uso di una griglia più fitta sarebbe stato praticamente impossibile. D'altra parte, considerando che la distanza fra i pilastri era di soli 6 metri (apparentemente trascurabile triangolo poteva sembrare più che sufficiente. Sfortunatamente la griglia usata era troppo rada per garantire una buona accuratezza dei risultati. Come conseguenza gli sforzi d itaglio che la struttura poteva sopportare furono sottostimati de $147 \%$, e questa sottostima influì pesantemente sulla realizzazione del progetto. furono costruiti sufficientemente spessi per 
sopp stimato in circa 700 milioni di dollari, il crollo d entità 3 sulla scala Richter.

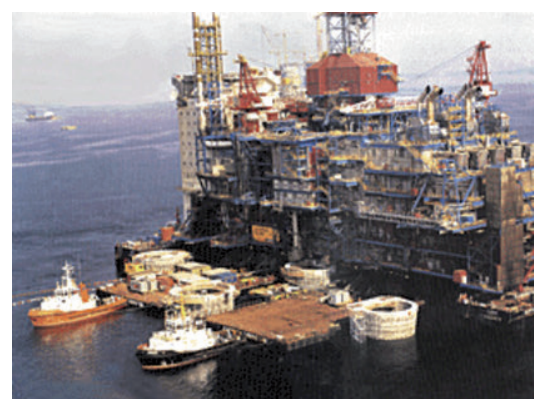

Fig. 12. Sleipner-A.

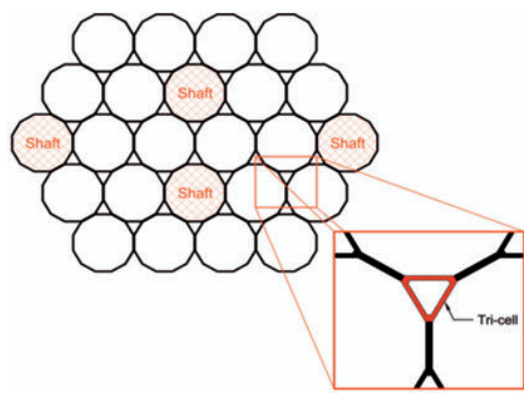

Fig. 13. Tri-cell.

\section{Il Ruolo del Matematico nel Calcolo Scientifico}

Quanto ho esposto finora non chiarisce forse quale sia il ruolo del Matematico applicato nel Calcolo scientifico, che potrebbe sembrare del tutto simile a quello dell'Ingegnere. In effetti la linea di confine tra le due discipline è molto sottile e in alcuni casi addirittura indistinguibile. Tuttavia l'approccio è spesso sostanzialmente diverso. Per l'Ingegnere la priorità è risolvere un problema, o eventualmente una classe di problemi tutti di tipo molto simile. Ad esempio, si vuole decidere la forma e la struttura di un oggetto (diciamo, l'ala di un aereo) cercando di raggiungere un compromesso ideale tra peso, costo, prestazioni, robustezza, eccetera. Questo problema viene in generale affrontato simulando il comportamento di molte possibili scelte in molte differenti situazioni. E per ogni scelta della struttura e della forma dell'ala, e del tipo di situazione (volo a regime, atterraggio, decollo, vento trasversale, etc) si cerca di capire, con una simulazione al calcolatore, quale sarebbe il comportamento dell'aereo con la scelta in questione. In questo caso si faranno moltissime simulazioni di moltissime ali possibili, ma tutte solo leggermente diverse l'una dall'altra: come dicevo, si devono risolvere numerosissimi problemi tutti di tipo molto simile. L'Ingegnere si aspetta che, in generale, il metodo o i metodi di discretizzazione e risoluzione che hanno dato predizioni attendibili su uno di questi problemi daranno predizioni attendibili anche per tutti i problemi simili. E solo se palesemente questo, per 
alcune tipologie, non accade cercherà di adattare (o a volte anche solo rabberciare) il metodo per farlo funzionare ancora. Nel fare questo userà soprattuto la sua grande conoscenza della natura fisica del problema, e cercherà di capire le caratteristiche fisiche dei problemi per i quali il metodo non funziona bene. Per contro l'obiettivo del matematico è spesso, in primo luogo, quello di tracciare con la massima precisione possibile la linea di demarcazione tra i problemi a cui un certo metodo pùo essere applicato con successo ed i problemi per $\mathrm{i}$ quali necessita una correzione. E nel fare questo la cosa più importante è l'incontro tra la struttura matematica del metodo e la struttura matematica del problema. Per valori molto diversi di certi parametri (diciamo: molto grandi, oppure molto piccoli) la struttura matematica del metodo potrebbe, in un certo qual senso, "sbilanciarsi", e mutare le proprie caratteristiche fondamentali. Sarà in questi casi che la correzione si rende necessaria, e la natura matematica della correzione da fare sarà resa evidente dalla comprensione della natura dello sbilanciamento. Per l'ingegnere il successo sarà un progetto di ala migliore di quanto realizzato finora. Per il matematico il successo sarà aver capito con precisione quando il metodo smette di essere affidabile, e cosa bisogna fare esattamente per rimetterlo in sesto. Per uno specifico problema, in generale, l'ingegnere avrà l'indubbio vantaggio di una molto maggiore comprensione della fisica coinvolta. Ma l'arma segreta del matematico applicato è spesso la capacità di riconoscere la stessa struttura matematica in problemi di natura fisica molto diversa. Questo gli permette di trasferire aspetti fondamentali della conoscen$z a$ da un dominio applicativo ad un altro, a volte totalmente differente, e di vedere quindi il problema sotto un punto di vista innovativo. Questo gli permette anche di esportare diagnosi e cure da un settore applicativo ad altri settori applicativi di natura molto diversa, ma con struttura matematica simile. Per quanto la cosa possa sembrare poco credibile ad un profano, equazioni quasi identiche descrivono il movimento di una navetta spaziale in un campo gravitazionale e quello di una particella carica elettricamente in un campo elettrico. $\mathrm{O}$ ancora, equazioni molto simili descrivono il moto di un fluido viscoso e la deformazione di un corpo elastico. Oppure la diffusione di un inquinante in un fiume e il movimento degli elettroni in un dispositivo semiconduttore.

Affido la conclusione di questo breve discorso a due grandi scienziati del passato e, tengo a precisare, nessuno dei due Matematico in 
senso stretto, ma entrambi convinti sostenitori dell'importanza della Matematica.

La filosofia naturale è scritta in questo grandissimo libro che continuamente ci sta aperto innanzi agli occhi, io dico l'universo, ma non si pùo intendere se prima non s'impara a intender la lingua e conoscer $i$ caratteri nei quali è scritto. Egli è scritto in lingua matematica, e $i$ caratteri son triangoli, cerchi e altre figure geometriche, senza i quali mezzi è impossibile a intenderne umanamente parola; senza questi è un aggirarsi vanamente per un oscuro labirinto

Il Saggiatore (1623), Galileo Galilei

Nessuna certezza delle scienze è dove non si pùo applicare una delle scienze matematiche.

Leonardo da Vinci 